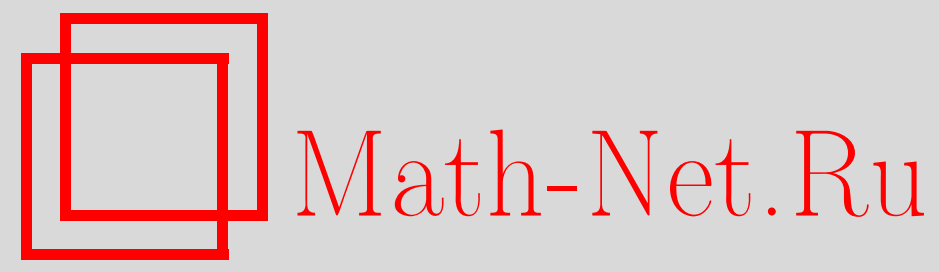

М. В. Болдин, Об оценке наименьших модулей в нестационарной авторегрессии и проверке стационарности, Теория вероятн. и ее примен., 1996, том 41, выпуск 2, 409-417

DOI: https://doi.org/10.4213/tvp2951

Использование Общероссийского математического портала Math-Net.Ru подразумевает, что вы прочитали и согласны с пользовательским соглашением http://www . mathnet.ru/rus/agreement

Параметры загрузки:

IP : 52.90 .164 .192

26 апреля 2023 г., 14:05:05

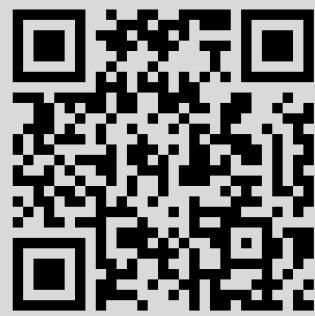


2. Вахания $H$. H. Вероятностные распределения в линейных пространствах. Тбилиси: Мецниереба, 1971, $156 \mathrm{c}$.

3. Вахания Н. Н., Тариеладзе В. И., Чобаяян С. А. Вероятностные распределения в банаховых пространствах. М.: Наука, 1985, 367 с.

4. Давыдов Ю. А., Лифиии М. А. Метод расслоений в некоторых вероятностных задачах. - Итоги науки и техники, сер. Теория вероятн. Матем. статист. Теорет. киберн., 1984, т. 22, с. 61-157.

5. Саханеяко А. И. Одна оценка для плотности распределения функционалов интегрального типа. - Труды Ин-та математики СО АН СССР, 1982, т. 1, с. 180-186.

6. Паулаускас В.И. О скорости сходимости в центральной предельной теореме в нехоторых банаховых пространствах. - Теория вероятн. и ее примен., 1976, т. XXI, в. 4, с. 775-791.

7. Паулаускас В.И. О плотности распределения нормы гауссовского вектора в банаховых пространствах. - Докл. АН СССР, 1982, т. 266, в. 6, с. 1301-1304.

8. Götze $F$. Asymptotic expansions for bivariate von Mises functionals. $-Z$. Wahrscheinlichkeitstheor. verw. Geb., 1979, B. 50, H. 3, S. 333-355.

9. Ehrhard A. Symmetrization dans l'espace de Gauss, - Math. Scand., 1983, v. 53, p. 281-301.

10. Kuelbs J., Kurtz T. Berry-Esseen estimates in Hilbert space and an application to the law of iterated logarithm. - Ann. Probab., 1974, v. 2, № 3, p. 287-407.

11. Rhee $W$., Talagrand $M$. Uniform convexity and distribution of the norm for Gaussian measure. - Probab. Theory Relat. Fields, 1986, v. 71, № 1, p. 59-64.

Поступила в редакцию

16.IV.1993

(C) $1996 \mathrm{r}$.

БОЛцин М. В.*

\section{ОБ ОЩЕНКЕ НАИМЕНЬШИХ МОДУЛЕЙ \\ В НЕСТАЦИОНАРНОЙ АВТОРЕГРЕССИИ И ПРОВЕРКЕ СТАЦИОНАРНОСТИ}

Для нестационарной схемы авторегрессии

$$
u_{i}=\beta_{i} u_{i-1}+\varepsilon_{i}, \quad \sup _{i}\left|\beta_{i}\right|<1,
$$

предлагаются тесты для проверки гипотезы стационарности $H_{0}: \beta_{i}=\beta, \beta$ неизвестно, основанные на процессе

$$
\widehat{w}_{n}(t)=\widehat{s}_{n}^{-1} n^{-1 / 2} \sum_{k=n t}^{[n t]} u_{k-1} \operatorname{sign}\left(u_{k}-\widehat{\beta}_{n, L D} u_{k-1}\right), \quad t \in[0,1],
$$

$\widehat{\beta}_{n, L D}$ - оценка наименьших модулей.

Ключевые слова и Фразы: авторегрессия, оценка нанменьших модулей, принцип инвариантности, близкие альтернативы.

*Mex.-матем. ф-т, кафедра теории вероятностей, МГУ, Воробьевы горы, 119899 Москва, Россия. 
1. Постановка задачи. Рассмотрим нестационарное соотношение авторегрессии с коэффициентом, зависящим от времени,

$$
u_{i}=\beta_{i} u_{i-1}+\varepsilon_{i}, \quad i \in \mathbf{Z}
$$

в котором $\left\{\beta_{i}\right\}$ - последовательность неслучайных неизвестных коэффициентов, $\sup _{i}\left|\beta_{i}\right| \leqslant b<1 ;\left\{\varepsilon_{i}\right\}$ - независимые одинаково распределенные случайные величины (н.о.р.сл.в.) с неизвестной функцией распределения $G(x)$. Будем предполагать, что

(i) $\mathbf{E} \varepsilon_{1}=0, \mathbf{E}\left|\varepsilon_{1}\right|^{2+\Delta}<\infty$ при некотором $\Delta>0$;

(ii) существует плотность $g(x)=G^{\prime}(x), \sup _{x} g(x)<\infty, g(x)$ удовлетворяет условию Липница в нуле, $g(0)>0$.

При условии (i) существует решение уравнения (1) вида

$$
u_{i}=\sum_{j \geqslant 0} \gamma_{i j} \varepsilon_{i-j},
$$

где $\gamma_{i 0}=1, \gamma_{i j}=\beta_{i} \beta_{i-1} \cdots \beta_{i-j+1}$ при $j \geqslant 1$, ряд сходится в $L^{2+\Delta}$.

Пусть наблюдения $u_{0}, u_{1}, \ldots, u_{n}$ удовлетворяют (2).

Цель работы состоит в построении тестов для проверки гипотезы стационарности

$$
H_{0}: \beta_{i}=\beta, \quad i \in \mathbf{Z}, \quad \beta \text { неизвестно, }
$$

и исследовании мощности тестов при альтернативе

$$
H_{1 n}: \beta_{k}=\beta+a_{k n} n^{-1 / 2}, \quad k=1, \ldots, n, \quad \sup _{k, n}\left|a_{k n}\right| \leqslant A<\infty .
$$

Тестовые статистики будут основываться на случайном процессе

$$
\widehat{w}_{n}(t)=\widehat{s}_{n}^{-1} n^{-1 / 2} \sum_{k=1}^{[n t]} u_{k-1} \operatorname{sign}\left(u_{k}-\widehat{\beta}_{n, L D} u_{k-1}\right), \quad t \dot{t} \in[0,1]
$$

где $\widehat{\beta}_{n, L D}$ - оценка наименьших модулей для $\beta$, построенная в предположении, что $H_{0}$ верна, $\widehat{s}_{n}^{2}=n^{-1} \sum_{k=1}^{n} u_{k}^{2},[\cdot]$ - целая часть числа

Похожие процессь использовались ранее в схемах линейной регрессии и авторегрессии (см. [1], [2]) для проверки гипотез о структуре схем.

2. Об оценке намменьших модулей. Если гипотеза $H_{0}$ верна, то в качестве оценки для $\beta$ можно взять оценку наименьших модулей $\widehat{\beta}_{n, L D}$, являюшуюся решением задачи

$$
\sum_{k=1}^{n}\left|u_{k}-\theta u_{k-1}\right| \longrightarrow \inf _{\theta \in \mathbb{R}^{1}} .
$$

Для непрерывной $G(x)$ решение (4) п.н. единственно и совпадает с одной из точек $u_{k} / u_{k-1}, k=1, \ldots, n$; оно может быть найдено либо перебором, либо более экономными способами (см., например, [3, раздел 1.1]).

Введем случайный процесс

$$
l_{n}(\theta)=\sum_{k=1}^{n} u_{k-1} \operatorname{sign}\left(u_{k}-\theta u_{k-1}\right), \quad \theta \in \mathbf{R}^{1} .
$$

Переписывая его в виде

$$
l_{n}(\theta)=\sum_{k=1}^{n}\left|u_{k-1}\right| \operatorname{sign}\left(u_{k} / u_{k-1}-\theta\right)
$$


видим, что траектории $l_{n}(\theta)$ - невозрастающие кусочно-постоянные функции со скачками в точках $u_{k} / u_{k-1}, k=1, \ldots, n$. Для непрерывной $G(x)$ решение задачи (4) совпадает с п.н. единственным решением уравнения

$$
l_{n}(\theta) \div 0
$$

(символ $\div$ в (5) означает переход через ноль), которое и будем дальше изучать.

Легко убедиться, что при условиях (i)-(ii) и справедливости $H_{0}$

$$
n^{-1} l_{n}(\theta)=\lambda(\theta)+o_{\mathbf{P}}(1), \quad n \rightarrow \infty,
$$

где

$$
\lambda(\theta)=-2 \mathbf{E}\left[u_{1}^{0} G\left((\theta-\beta) u_{1}^{0}\right)\right], \quad u_{i}^{0}=\sum_{j \geqslant 0} \beta^{j} \varepsilon_{i-j}
$$

и существует производная

$$
\lambda^{\prime}(\beta)=-2 g(0) \mathbf{E}\left(u_{1}^{0}\right)^{2}=-2 g(0) \mathbf{E} \varepsilon_{1}^{2}\left(1-\beta^{2}\right)^{-1} .
$$

Обозначим $a_{n}(t)=\sum_{k=1}^{[n t]} a_{k n}, t \in[0,1]$.

Следуюшая теорема 1 дает равномерное линейное стохастическое разложение процесса $n^{-1 / 2} l_{n}(\theta)$ в окрестности $\beta$, справедливое при $H_{1 n}$.

Теорема 1. Пусть выполнены условия (i)-(ii), и верна альтернатива $H_{1 n}$. Тогда при $n \rightarrow \infty$ и пюбом $0<\Theta<\infty$

$$
\sup _{|\theta| \leqslant \Theta}\left|n^{-1 / 2} l_{n}\left(\beta+\theta n^{-1 / 2}\right)-n^{-1 / 2} \sum_{k=1}^{n} u_{k-1}^{0} \operatorname{sign} \varepsilon_{k}-\lambda^{\prime}(\beta)\left[\theta-a_{n}(1)\right]\right|=o_{\mathbf{P}}(1)
$$

Теорема 1 будет доказана в п. 4. Она легко влечет за собой следующее утверждение.

Следствие 1. Если өыполнены условия (i)-(ii), верна альтернатива $H_{1 n}$ u $\sqrt{n}\left(\widehat{\beta}_{n}-\beta\right)=O_{\mathbf{P}}(1)$, mo npu $n \rightarrow \infty$

$$
n^{-1 / 2} l_{n}\left(\widehat{\beta}_{n}\right)=n^{-1 / 2} \sum_{k=1}^{n} u_{k-1}^{0} \operatorname{sign} \varepsilon_{k}+\lambda^{\prime}(\beta)\left[\sqrt{n}\left(\widehat{\beta}_{n}-\beta\right)-a_{n}(1)\right]+o_{P}(1) .
$$

В силу теоремы 21.1 [4] (см. также пример 1 в $\$ 21[4]$ )

$$
n^{-1 / 2} \sum_{k=1}^{n} u_{k-1}^{0} \operatorname{sign} \varepsilon_{k} \longrightarrow \mathcal{N}\left(0, \mathbf{E}\left(u_{1}^{0}\right)^{2}\right), \quad n \rightarrow \infty
$$

Последнее замечание и следствие 1 влекут за собой теорему 2.

Теорема 2. Пусть өьполнены условия (i)-(ii). Тогда, если верка $H_{1 n}$, то дяя решения $\widehat{\beta}_{n, L D}$ уравнения (5) справедливо представление

$$
\sqrt{n}\left(\widehat{\beta}_{n, L D}-\beta\right)=-\left[\lambda^{\prime}(\beta)\right]^{-1} n^{-1 / 2} \sum_{k=1}^{n} u_{k-1}^{0} \operatorname{sign} \varepsilon_{k}+a_{n}(1)+o_{\mathbf{P}}(1)
$$

$u$, следовательно, при $a_{n}(1) \rightarrow a(1)$

$$
\sqrt{n}\left(\widehat{\beta}_{n, L D}-\beta\right) \longrightarrow \mathcal{N}\left(a(1), \frac{1-\beta^{2}}{[2 g(0)]^{2} \mathrm{E} \varepsilon_{1}^{2}}\right), \quad n \rightarrow \infty .
$$

Теорема 2 будет доказана в п. 5. 
При $H_{0}$ асимптотический сдвиг $a(1)=0$ и утверждение теоремы 2 совпадает с известным утверждением об асимптотической нормальности оценки наименьших модулей в стационарной схеме авторегрессии (см., например, [5]). Подчеркнем, что наш подход и в стационарном случае отличается от использованного в [5]:

3. Предельные распределения $\widehat{w}_{n}(t)$ и тестовых статистик. Введем случайный процесс

$$
w_{n}(t)=\left[\mathbf{E}\left(u_{1}^{0}\right)^{2}\right]^{-1 / 2} n^{-1 / 2} \sum_{k=1}^{[n t]} u_{k-1} \operatorname{sign}\left(u_{k}-\widehat{\beta}_{n, L} D^{u_{k-1}}\right) ; \quad t \in[0,1] .
$$

Процесс $w_{n}(t)$ построен «в соответствии» с уравнением (5), определяющим $\widehat{\beta}_{n, L D}$, в частности, $w_{n}(1)=\left[\mathrm{E}\left(u_{1}^{0}\right)^{2}\right]^{-1 / 2} n^{-1 / 2} l_{n}\left(\widehat{\beta}_{n, L D}\right)$.

Пусть $w(t)$ - броуновский мост, а $\mathrm{D}[0,1]$ - метрическое пространство Скорохода $[4$, гл. 5]. Будем предполагать, что для некоторой функции $a(t) \in \mathbf{D}[0,1]$

$$
\sup _{t}\left|a_{n}(t)-a(t)\right|=o(1), \quad n \rightarrow \infty
$$

Теорема 3. Пусть выполнены условия (i)-(ii). Тогда при аипотезе $H_{0}$ u $n \rightarrow$ $\infty$ прочесс $w_{n}(t)$ слабо сходится в $\mathrm{D}[0,1] \kappa w(t)$, а при альтернативе $H_{1 n}$ и добавочном услович (iii) $-\kappa w(t)+\sigma(\beta) \delta(t)$, где $\delta(t)=a(t)-t a(1)$,

$$
\sigma(\beta)=2 g(0)\left(\mathbf{E} \varepsilon_{1}^{2}\right)^{1 / 2}\left(1-\beta^{2}\right)^{-1 / 2} .
$$

Схема доказательства теоремы 3 будет приведена в п. 6.

Пусть процесс $\widehat{w}_{n}(t)$ определен в (3). Поскольку $\widehat{s}_{n}^{2}-$ состоятельная $H_{1 n}$ оценка $\mathbf{E}\left(u_{1}^{0}\right)^{2}$, то для $\widehat{w}_{n}(t)$ справедливы те же утверждения о слабой сходимости, что и для $w_{n}(t)$. Поэтому в условиях теоремы 3 статистихи

$$
\begin{aligned}
& \widehat{D}_{n}=\sup _{t}\left|\widehat{w}_{n}(t)\right|=\widehat{s}_{n}^{-1} \max _{m \leqslant n}\left|n^{-1 / 2} \sum_{k=1}^{m} u_{k-1} \operatorname{sign}\left(u_{k}-\widehat{\beta}_{n, L D} u_{k-1}\right)\right| \\
& \widehat{\omega}_{n}^{2}=\widehat{s}_{n}^{-2} \int_{0}^{1} \widehat{w}_{n}^{2}(t) d t=\widehat{s}_{n}^{-2} n^{-2} \sum_{m=1}^{n}\left[\sum_{k=1}^{m} u_{k-1} \operatorname{sign}\left(u_{k}-\widehat{\beta}_{n, L D} u_{k-1}\right)\right]
\end{aligned}
$$

сходятся по распределению к $\sup _{t}|w(t)|$ и $\int_{0}^{1} w^{2}(t) d t$.

Распределения последних - известные распределения А. Н. Колмогорова и Н. В. Смирнова. Статистики $\widehat{D}_{n}$ и $\widehat{\omega}_{n}^{2}-$ статистики тестов для проверки $\boldsymbol{H}_{0}$. При альтернативе $H_{1 n}$ они сходятся соответственно $\sup _{t}|w(t)+\sigma(\beta) \delta(t)|$ и $\int_{0}^{1}[w(t)+$ $\sigma(\beta) \delta(t)]^{2} d t$.

Строить случайные процессы типа $\widehat{w}_{n}(t)$ можно, разумеется, не только с помощью $\widehat{\beta}_{n, L D}$, но и других М-оценок. Необходимо лишь, чтобы структура процесса была «согласована» с уравнением, определяющим оценку. Например, для оценки наименьших квадратов $\widehat{\beta}_{n, L S}$, определяемой уравнением

$$
\sum_{k=1}^{n} u_{k-1}\left(u_{k}-\theta u_{k-1}\right)=0
$$

можно рассмотреть процесс

$$
\widehat{w}_{n}^{L S}(t)=\widehat{s}_{n, L S}^{-1} n^{-1 / 2} \sum_{k=1}^{[n t]} u_{k-1}\left(u_{k}-\widehat{\beta}_{n, L S} u_{k-1}\right)
$$


где $\widehat{s}_{n, L S}^{2}-$ состоятельная оценка $\mathrm{E}\left(u_{1}^{0}\right)^{2} \mathrm{E} \varepsilon_{1}^{2}$. При $H_{1 n}$ и (i) он будет сходиться $\mathbf{x}$

$$
w(t)+\sigma_{L S}(\beta) \delta(t), \quad \sigma_{L S}(\beta)=\left(1-\beta^{2}\right)^{-1 / 2} .
$$

Отметим особо, что предположение $\mathbf{E}\left|\varepsilon_{1}\right|^{2+\Delta}<\infty$ вовсе не является существенным для нашего подхода и определяется лишь используемой оценкой. При $H_{0}$ в случае $\mathbf{E} \varepsilon_{1}^{2}=\infty$ не выполнены достаточные условия локальной асимптотической нормальности (ЛАН) для наблюдений (о достаточных условиях ЛАН см. [6]), и общеупотребительные оценки, в частности, $\widehat{\beta}_{n, L D}$, перестают быть асимптотически нормальными [3, Sect. 3.2, 3.4], [5]. В этом случае можно использовать, например, медианную оценку из [2], которая асимптотически нормальна при $E\left|\varepsilon_{1}\right|^{1+\Delta}<\infty$.

4. Доказательство теоремы 1. Пусть $\Delta_{k}(x)$ равняется $1, \frac{1}{2}$ или 0 при $\varepsilon_{k}<x$, $\varepsilon_{k}=x, \varepsilon_{k}>x$ соответственно. Тогда

$$
n^{-1 / 2} l_{n}\left(\beta+\theta n^{-1 / 2}\right)-n^{-1 / 2} \sum_{k=1}^{n} u_{k-1} \operatorname{sign} \varepsilon_{k}=-2 z_{1 n}(\theta)-2 z_{2 n}(\theta)
$$

rде

$$
\begin{aligned}
z_{1 n}(\theta)=n^{-1 / 2} \sum_{k=1}^{n} u_{k-1}[ & {\left[\Delta_{k}\left(\left(\theta-a_{k n}\right) u_{k-1} n^{-1 / 2}\right)\right.} \\
& \left.-G\left(\left(\theta-a_{k n}\right) u_{k-1} n^{-1 / 2}\right)-\Delta_{k}(0)+G(0)\right] \\
z_{2 n}(\theta)=n^{-1 / 2} \sum_{k=1}^{n} u_{k-1} & {\left[G\left(\left(\theta-a_{k n}\right) u_{k-1} n^{-1 / 2}\right)-G(0)\right] }
\end{aligned}
$$

Лемма 1. При условияx (i)-(ii)

$$
\sup _{|\theta| \leqslant \Theta}\left|z_{1 n}(\theta)\right|=o_{\mathbf{P}}(1), \quad n \rightarrow \infty
$$

Д о к а 3 а т е ль с т в о. Представим $z_{1 n}(\theta)$ в виде

$$
z_{1 n}(\theta)=p_{1 n}(\theta)+p_{2 n}(\theta)
$$

где

$$
\begin{aligned}
p_{1 n}(\theta)= & n^{-1 / 2} \sum_{k=1}^{n} u_{k-1} I\left\{u_{k-1}>0\right\} \\
& \times\left[\Delta_{k}\left(\left(\theta-a_{k n}\right) u_{k-1} n^{-1 / 2}\right)-G\left(\left(\theta-a_{k n}\right) u_{k-1} n^{-1 / 2}\right)-\Delta_{k}(0)+G(0)\right]
\end{aligned}
$$

$I(\cdot)$ - индикатор события, а $p_{2 n}(\theta)$ определяется аналогичным выражением, в котором $I\left\{u_{k-1}>0\right\}$ заменен на $I\left\{u_{k-1} \leqslant 0\right\}$.

Разделим отрезок $[-\Theta, \Theta]$ на $3^{m_{n}}$ частей точками $\eta_{a n}=-\Theta+2 \Theta 3^{-m_{n} s, s=}$ $0,1, \ldots, 3^{m_{n}}$, к пусть $3^{m_{n}} \theta n^{\delta / 4}$ при $n \rightarrow \infty$, где $\delta=\min (\Delta, 1)$. Пусть $\eta_{j n}-$ та точка среди $\left\{\eta_{s n}\right\}$, для которой

$$
0 \leqslant \eta_{j n}-\theta \leqslant 2 \Theta 3^{-m_{n}} .
$$

Обозначим

$$
\begin{aligned}
& \widehat{u}_{k-1, s}=u_{k-1}\left[1-2 \Theta 3^{-m_{n}} \eta_{s n}^{-1} I\left\{u_{k-1} \leqslant 0\right\}\right] \\
& \tilde{u}_{k-1, s}=u_{k-1}\left[1-2 \Theta 3^{-m_{n}} \eta_{s n}^{-1} I\left\{u_{k-1}>0\right\}\right]
\end{aligned}
$$


$k=1, \ldots, n, s=0,1, \ldots, 3^{m_{n}}$. Для построенных величин в силу (9) и определений (10) и (11) справедливы неравенства

$$
\eta_{j n} \widetilde{u}_{k-1, j} \leqslant \theta u_{k-1} \leqslant \eta_{j n} \widehat{u}_{k-1, j}, \quad k=1, \ldots, n .
$$

Обозначим

$$
\begin{aligned}
\widehat{U}_{s n}= & \left(\widehat{u}_{0 s}, \ldots, \widehat{u}_{n-1, s}\right), \quad \widetilde{U}_{s n}=\left(\widetilde{u}_{0 s}, \ldots, \widetilde{u}_{n-1, \Delta}\right) \\
q_{n}\left(\eta_{j n}, \widehat{U}_{j n}\right)= & n^{-1 / 2} \sum_{k=1}^{n} u_{k-1} I\left\{u_{k-1}>0\right\} \\
& \times\left[\Delta_{k}\left(\left(\eta_{j n} \widehat{u}_{k-1, j}-a_{k n} u_{k-1}\right) n^{-1 / 2}\right)\right. \\
& \left.-G\left(\left(\eta_{j n} \widehat{u}_{k-1, j}-a_{k n} u_{k-1}\right) n^{-1 / 2}\right)-\Delta_{k}(0)+G(0)\right] .
\end{aligned}
$$

В силу монотонности по $y$ функций $\Delta_{k}(y), G(y)$ и соотношения (12), справедливы неравенства

$$
\begin{aligned}
p_{1 n}(\theta) \leqslant & q_{n}\left(\eta_{j n}, \widehat{U}_{j n}\right)+n^{-1 / 2} \sum_{k=1}^{n} u_{k-1} I\left\{u_{k-1}>0\right\} \\
& \times\left[G\left(\left(\eta_{j n} \widehat{u}_{k-1, j}-a_{k n} u_{k-1}\right) n^{-1 / 2}\right)-G\left(\left(\eta_{j n} \widetilde{u}_{k-1, j}-a_{k n} u_{k-1}\right) n^{-1 / 2}\right)\right], \\
p_{1 n}(\theta) \geqslant & q_{n}\left(\eta_{j n}, \widetilde{U}_{j n}\right)-n^{-1 / 2} \sum_{k=1}^{n} u_{k-1} I\left\{u_{k-1}>0\right\} \\
& \times\left[G\left(\left(\eta_{j n} \widehat{u}_{k-1, j}-a_{k n} u_{k-1}\right) n^{-1 / 2}\right)-G\left(\left(\eta_{j n} \widetilde{u}_{k-1, j}-a_{k n} u_{k-1}\right) n^{-1 / 2}\right)\right] .
\end{aligned}
$$

Следовательно,

$$
\begin{aligned}
& \sup _{|\theta| \leqslant \Theta}\left|p_{1 n}(\theta)\right| \leqslant \sup _{0 \leqslant j \leqslant 3^{m}}\left\{\left|q_{n}\left(\eta_{j n}, \widehat{U}_{j n}\right)\right|+\left|q_{n}\left(\eta_{j n}, \widetilde{U}_{j n}\right)\right|\right\} \\
& +\sup _{0 \leqslant j \leqslant 3_{n}} n^{-1 / 2} \sum_{k=1}^{n} u_{k-1} I\left\{u_{k-1}>0\right\} \\
& \quad \times\left[G\left(\left(\eta_{j n} \widehat{u}_{k-1, j}-a_{k n} u_{k-1}\right) n^{-1 / 2}\right)-G\left(\left(\eta_{j n} \widetilde{u}_{k-1, j}-a_{k n} u_{k-1}\right) n^{-1 / 2}\right)\right] .
\end{aligned}
$$

С помощью формулы Тейлора, условий (i)-(ii) и определений (10), (11) убеждаемся, что второе слагаемое в правой части (13) есть $O_{\mathbf{P}}\left(3^{-m_{n}}\right)=o_{\mathbf{P}}(1), n \rightarrow \infty$.

Рассмотрим В (13) $q_{n}\left(\eta_{j n}, \widehat{U}_{j n}\right)$. Положим для краткости

$$
\begin{aligned}
\xi_{k}= & \xi_{k}(j, n):=u_{k-1} I\left\{u_{k-1}>0\right\} \\
\times & {\left[\Delta_{k}\left(\left(\eta_{j n} \widehat{u}_{k-1, j}-a_{k n} u_{k-1}\right) n^{-1 / 2}\right)\right.} \\
& \left.\quad-G\left(\left(\eta_{j n} \widehat{u}_{k-1, j}-a_{k n} u_{k-1}\right) n^{-1 / 2}\right)-\Delta_{k}(0)+G(0)\right] .
\end{aligned}
$$

Пусть $\Omega_{\leqslant i}-$ сигма-алгебра, порожденная $\left\{\varepsilon_{\theta}, s \leqslant i\right\}$. Очевидно,

$$
\mathbf{E} \xi_{k}=\mathbf{E}\left[\mathbf{E}\left(\xi_{k} \mid \Omega_{\leqslant k-1}\right)\right]=0,
$$


так хак $\mathbf{E}\left(\xi_{k} \mid \Omega_{\leqslant k-1}\right)=0$ п.н.

Аналогично, для $k<j$

$$
\mathbf{E} \xi_{k} \xi_{j}=\mathbf{E}\left[\mathbf{E}\left(\xi_{k} \xi_{j} \mid \Omega_{\leqslant j-1}\right)\right]=\mathbf{E}\left[\xi_{k} \mathbf{E}\left(\xi_{j} \mid \Omega_{\leqslant j-1}\right)\right]=0 .
$$

Кроме того, $\forall x_{1}, x_{2} \in \mathbf{R}^{1}$ и $\delta=\min (\Delta, 1)$

$$
\mathbf{E}\left[\Delta_{k}\left(x_{1}\right)-G\left(x_{1}\right)-\Delta_{k}\left(x_{2}\right)+G\left(x_{2}\right)\right]^{2} \leqslant\left|G\left(x_{1}\right)-G\left(x_{2}\right)\right| \leqslant\left|G\left(x_{1}\right)-G\left(x_{2}\right)\right|^{\delta} .
$$

Отсода $\mathbf{E} \xi_{k}^{2} \leqslant \mathbf{E} u_{k-1}^{2}\left|G\left(\eta_{j n} \widehat{u}_{k-1, j}-a_{k n} u_{k-1}\right) n^{-1 / 2}-G(0)\right|^{\delta}=O\left(n^{-\delta / 2}\right)$ равномерно по $k, j$ в силу (i), (ii), $\sup _{k, n}\left|a_{k n}\right| \leqslant A$ и неравенства $\left|\widehat{u}_{k-1, j}\right| \leqslant 3\left|u_{k-1}\right|$, следующего из (10).

Используя равенство $q_{n}\left(\eta_{j n}, \widehat{U}_{j n}\right)=n^{-1 / 2} \sum_{k=1}^{n} \xi_{k}$, находим:

$$
\sup _{0 \leqslant j \leqslant 3_{n}} \mathbf{E} q_{n}^{2}\left(\eta_{j n}, \widehat{U}_{j n}\right)=O\left(n^{-\delta / 2}\right) .
$$

Теперь из (14) $\forall \varepsilon>0$ в силу неравенства Чебышева

$$
\begin{aligned}
& \mathbf{P}\left\{\sup _{0 \leqslant j \leqslant 3^{m_{n}}}\left|q_{n}\left(\eta_{j n}, \widehat{U}_{j n}\right)\right|>\varepsilon\right\} \leqslant \sum_{j=0}^{3^{m_{n}}} \mathbf{P}\left\{\left|q_{n}\left(\eta_{j n}, \widehat{U}_{j n}\right)\right|>\varepsilon\right\} \\
& \quad \leqslant \varepsilon^{-2} \sum_{j=0}^{3^{m_{n}}} \mathbf{E} q_{n}^{2}\left(\eta_{j n}, \widehat{U}_{j n}\right)=O\left(3^{m_{n} n^{-\delta / 2}}\right)=O\left(n^{-\delta / 4}\right)=o(1), \quad n \rightarrow \infty,
\end{aligned}
$$

в силу выбора $m_{n}$.

Аналогично показывается, что

$$
\sup _{0 \leqslant j \leqslant 3^{m_{n}}}\left|q_{n}\left(\eta_{j n}, \tilde{U}_{j n}\right)\right|=o_{\mathbf{P}}(1), \quad n \rightarrow \infty
$$

Следовательно, первое слагаемое в правой части (13) есть о $\mathbf{P}(1), n \rightarrow \infty$. Значит,

$$
\sup _{|\theta| \leqslant \Theta}\left|p_{2 n}(\theta)\right|=o_{P}(1), \quad n \rightarrow \infty .
$$

Аналогично похазывается, что

$$
\sup _{|\theta| \leqslant \Theta}\left|p_{2 n}(\theta)\right|=o_{P}(1), \quad n \rightarrow \infty .
$$

Лемма 1 доказана.

Лемма 2. При условиях (i)-(ii)

$$
\sup _{|\theta| \leqslant \Theta}\left|z_{2 n}(\theta)-g(0)\left(\theta-a_{n}(1)\right) \mathbf{E} \varepsilon_{1}^{2}\left(1-\beta^{2}\right)^{-1}\right|=o_{\mathbf{P}}(1), \quad n \rightarrow \infty .
$$

Утверждение леммы 2 следует из формулы Тейлора и закона больших чисел.

Утверждение теоремы 1 следует теперь из (8), лемм 1,2 и сходимости

$$
n^{-1 / 2} \sum_{k=1}^{n} u_{k-1} \operatorname{sign} \varepsilon_{k}-n^{-1 / 2} \sum_{k=1}^{n} u_{k-1}^{0} \operatorname{sign} \varepsilon_{k}=o_{P}(1), \quad n \rightarrow \infty
$$

5. Доказательство теоремы 2. В силу теоремы 1 и соотношения (7) при достаточно большом $B>0$ выполнено неравенство $\ln _{n}\left(\beta-B n^{-1 / 2}\right)>0$ с вероятностью, сколь угодно близкой к единице, равномерно по $n>n_{0}$ и, аналогично, 
$l_{n}\left(\beta-B n^{-1 / 2}\right)<0$. Это влечет за собой, в силу монотонности $l_{n}(\theta)$, соотношение $\widehat{\beta}_{n, L D} \in\left[\beta-B n^{-1 / 2}, \beta+B n^{-1 / 2}\right]$ с вероятностьк, сколь угодно близхой $\mathrm{x}$ единице, равномерно по $n>n_{0}$. Следовательно,

$$
\sqrt{n}\left(\hat{\beta}_{n, L D}-\beta\right)=O_{\mathbf{P}}(1), \quad n \rightarrow \infty
$$

Теперь отметим, что

$$
n^{-1 / 2} l_{n}\left(\bar{\beta}_{n, L D}\right)=o_{P}(1), \quad n \rightarrow \infty
$$

Действительно, схачки функции $l_{n}(\theta)$ не больше $\max _{1 \leqslant k \leqslant n}\left|u_{k-1}\right| \leqslant \max _{0 \leqslant k \leqslant n-1}\left|\varepsilon_{k}\right|$ $\times(1-b)^{-1}+\left|u_{0}\right|$. Поскольку при $\mathbf{E} \varepsilon_{1}^{2}<\infty$

$$
n^{-1 / 2} \max _{0 \leqslant k \leqslant n-1}\left|\varepsilon_{k}\right|=o_{\mathbf{P}}(1),
$$

To

$$
n^{-1 / 2} \max _{1 \leqslant k \leqslant n}\left|u_{k-1}\right|=o_{P}(1), \quad n \rightarrow \infty .
$$

Теперь остается положить в (6) $\widehat{\beta}_{n}=\widehat{\beta}_{n, L D}$ и разрешить (6) относительно $\sqrt{n}\left(\widehat{\beta}_{n, L D}-\beta\right)$. Теорема 2 доказана.

6. Доказательство теоремы 3. Приведем схему доказательства в предположении справедливости $H_{1 n}$.

Справедливо следующее соотношение, доказываемое аналогично теореме 1: при любом $0<\Theta<\infty$ и $n \rightarrow \infty$ в условиях теоремы 3

$$
\begin{aligned}
\sup _{|\theta| \leqslant \Theta 1 \leqslant m \leqslant n} \sup _{1 \leqslant m} \mid n^{-1 / 2} \sum_{k=1}^{m} u_{k-1} \operatorname{sign}\left[u_{k}-\left(\beta+\theta n^{-1 / 2}\right) u_{k-1}\right] \\
-n^{-1 / 2} \sum_{k=1}^{m} u_{k-1}^{0} \operatorname{sign} \varepsilon_{k}-\lambda^{\prime}(\beta) \theta m / n \\
+\lambda^{\prime}(\beta) n^{-1} \sum_{k=1}^{m} a_{k n} \mid=o_{\mathrm{P}}(1)
\end{aligned}
$$

Из (15) аналогично следствию 1 получаем, что для любой последовательности $\widehat{\beta}_{n}$ такой, что $\sqrt{n}\left(\widehat{\beta}_{n}-\beta\right)=O_{\mathbf{P}}(1)$, имеет место соотношение

$$
\begin{aligned}
\sup _{1 \leqslant m \leqslant n} \mid n^{-1 / 2} \sum_{k=1}^{m} u_{k-1} \operatorname{sign}\left(u_{k}-\widehat{\beta}_{n} u_{k-1}\right)-n^{-1 / 2} \sum_{k=1}^{m} u_{k-1}^{0} \operatorname{sign} \varepsilon_{k} & \\
& -\lambda^{\prime}(\beta) \sqrt{n}\left(\widehat{\beta}_{n}-\beta\right) m / n+\lambda^{\prime}(\beta) n^{-1} \sum_{k=1}^{m} a_{k n} \mid=\operatorname{oP}_{\mathrm{P}}(1), \quad n \rightarrow \infty
\end{aligned}
$$

Обозначим

$$
\nu_{n}(t)=\left[\mathrm{E}\left(u_{1}^{0}\right)^{2}\right]^{-1 / 2} n^{-1 / 2} \sum_{k=1}^{[n t]} u_{k-1}^{0} \operatorname{sign} \varepsilon_{k} .
$$

В силу теоремы 2 при $H_{1 n}$ и $n \rightarrow \infty$

$$
\sqrt{n}\left(\widehat{\beta}_{n, L D}-\beta\right)=\sigma^{-1}(\beta) \nu_{n}(1)+a_{n}(1)+o_{P}(1), \quad n \rightarrow \infty
$$

Следовательно,

$$
\sqrt{n}\left(\hat{\beta}_{n, L D}-\beta\right)=O_{\mathrm{P}}(1)
$$


Отсюда и из (16) при $\widehat{\beta}_{n}=\widehat{\beta}_{n, L D}$ несложно получить, что

$$
\sup _{0 \leqslant t \leqslant 1}\left|w_{n}(t)-\nu_{n}(t)-\sigma(\beta) \sqrt{n}\left(\widehat{\beta}_{n, L D}-\beta\right) t-\sigma(\beta) a_{n}(t)\right|=o_{\mathbf{P}}(1), \quad n \rightarrow \infty .
$$

Подставляя (17) в (18), находим, что при $n \rightarrow \infty$

$$
\sup _{0 \leqslant t \leqslant 1}\left|w_{n}(t)-\left[\nu_{n}(t)-t \nu_{n}(1)\right]-\sigma(\beta)\left[a_{n}(t)-t a_{n}(1)\right]\right|=o_{\mathbf{P}}(1) .
$$

В силу теоремы 21.1 из [4] и примера 1 в $\$ 21$ [4], $\nu_{n}(t)$ при $n \rightarrow \infty$ слабо сходится в $\mathrm{D}[0,1]$ х стандартному винеровскому процессу $\nu(t)$. Следовательно, $\nu_{n}(t)-t \nu_{n}(1)$ сходится к броуновскому мосту $w(t)=\nu(t)-t \nu(1)$, что совместно с (19) доказывает теорему 3.

В заключение автор выражает благодарность профессору Ю. Н. Тюрину за полезное обсуждение работы.

\section{СПИСОК ЛИТЕРАТУРЫ}

1. Болдия M. B. О распределении статистик серий в схеме линейной регрессии. Теория вероятн. и ее примен., 1993, т. 38, в. 1, с. 154-161.

2. Boldin $M$. V. On median tests and estimates in autoregressive models. - Math. Methods Statist., 1994, v. 3, № 2, p. 114-129.

3. Bloomfield P., Steiger W. Least absolute deviations. - Boston: Birkhäuser, 1983, $349 \mathrm{p}$.

4. Биллияасли II. Сходимость вероятностных мер. М.: Наука, 1977, 352 с.

5. Pollard $D$. Asymptotics for least absolute deviation regression estimators. - Econometric Theory, 1991, v. 7, p. 186-199.

6. Kreiss J.-P. On adaptive estimation in stationary ARMA-processes. - Ann. Statist., 1987 , v. 15 , № 1, p. 112-123.

\section{(C) 1996 r. ГОРЯйнОВ В. В.*, ПОЛКОВникоВ А. А.**}

\section{О ПРЕДЕЛЬНЫХ РАСПРЕДЕЛЕНИЯХ ВЕРОЯТНОСТЕЙ ДЛЯ ДОКРИТИЧЕСКИХ ВЕТВЯЩИХСЯ ПРОЦЕССОВ}

Изучаются свойства распределений вероятностей, возникаюших в предельной теореме для докритических ветвяшихся процессов. Получено описание предельных распределений для процессов с непрерывным временем и изучен вопрос сушествования и построения процессов, имеюших предельное распределение с заданными начальными вероятностями.

Ключевые слова $и$ фразы: докритический ветвящийся процесс, инфинитезимальная пронзводяая функция, предельное распределение, предельная интерполяционная задача.

*Институт прикладной математики и механики АН Украины, Донецк, Украина. ** Донецкий государственный университет, Донецк, Укранна. 